\title{
Design of Automatic Testing System for Steel Tape Based on Image Processing
}

\author{
Zhao Baoya \\ North China Institute of Aerospace Engineering \\ Precision grating displacement sensor and Detection \\ Technology Engineering Laboratory of Hebei Province \\ Langfang, Hebei, China, 13930663584 \\ Baoya_yg@126.com \\ Kang Huifeng \\ North China Institute of Aerospace Engineering \\ Precision grating displacement sensor and Detection \\ Technology Engineering Laboratory of Hebei Province \\ Langfang, Hebei, China
}

\begin{abstract}
Refer to the problems about the methods of steel tape calibration with hard labor work and low efficiency, it has been dealt with new calibration method based on digital image processing, which is used CCD camera to collect images of the steel tape standard at every meter, then modify the images with specified software which is developed by Visual Basic 6.0. it is described the whole system constructions and the methods to achieve, meanwhile, The methods of Image processing are introduced in details. The results demonstrated that this system realized automatic testing for steel tape by the verification regulation. It can improve efficiency and precision.
\end{abstract}

Keywords-digital image processing; steel tape; automatic verification system

\section{INTRODUCTION}

The Computer Vision Technology has been widely used in industrial quality calibration/inspection field because of its significant advantages with non-contact, high speed, moderate precision and achieved inspection on line, etc.

The steel tape must be calibration timely during its usage, because its precision will serious affect the measurement precision. At present, most metrological agencies calibrate the steel tape manually, comparing the error between the tested steel tape and the standard steel tape with microscope, this method is the low degree of automation, digitalization with hard labor work.

So it becomes urgently to realize an automatic system which can take place of the old methods to improve efficiency. Based on the steel tape measurement regulation, using CCD image processing automatic system and Visual Basic 6 image modification software, we can deal with it automatically.The

\author{
Wei Caiqiao \\ North China Institute of Aerospace Engineering \\ Precision grating displacement sensor and Detection \\ Technology Engineering Laboratory of Hebei Province \\ Langfang, Hebei, China \\ wcq887351@sina.com \\ Yang Gang \\ Siteco Prosperity Lighting (Langfang) Co., Ltd. \\ Langfang, Hebei, China, 13582665663 \\ 97131.yang@sohu.com
}

inspection results will be output with high precision less than 12 micra. It is operated easily. For the $10 \mathrm{~m}$ longer ones, it can be segmented calibrated.

\section{IMAGE ACQUISITION SYSTEM}

The image acquisition system is composed of computer, single-chip computer, stepping motor, stepping motor driver, $10 \mathrm{~m}$ test-platform, industrial cameras and sensors, etc.

To input control commands through the computer software interface, under the control of the computer and single-chip microcomputer to complete detection of locomotive operation, image acquisition, image processing identification and inspection report printing,etc.

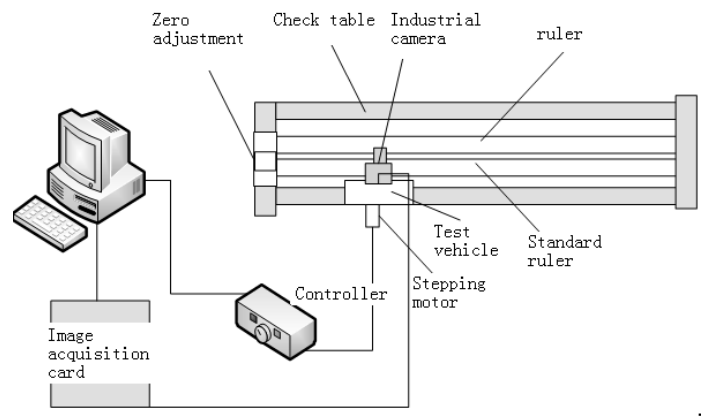

Fig 1 Steel tape detection system

When we calibrating the steel tape, we place the standard steel tape on the long inspection table, steel tape zero by zero adjustment positioning and clamping tightly, the other end loading specified in the verification regulation of weights, then inspected tape is in juxtaposed position and fixed on the work table, the other end with weights adjusting to zero screw, then zero is adjusted.

In the computer software system, we input detection

This work is supported by Hall of Hebei province science and technology( code: 11213569),

Langfang city science and Technology Bureau ～(code: 2011011025) 
information and control command, through the computer serial port output control commands and associated parameters to the controller of SCM, SCM will receive the string parsing, extract the control command, according to the command output the given frequency of the pulse signal and direction signal to the stepper motor drive, driven by a stepper motor inspection car running, computer controlled slider is moved to each test location by the single chip microcomputer, CCD camera by computer control uptake of standard steel tape and the tested steel tape line imaged, after the high speed real time graphics card collection is converted into a digital image, and enter the computer digital image processing. System image processing gray, binary processing, edge extraction processing and linear search, the standard steel tapes and the tested steel tape detection line coordinate values are compared, difference and obtained length and the pixel value and corresponding treatment, the results output to test the original records, and automatically make the file backup. For the $10 \mathrm{~m}$ longer steel tape, it will be used segmentation methods for end-to-end testing.

\section{DESIGN CONTROL SYSTEM}

Single-chip microcomputer is the core of Control system, through the serial communication between PC microcontroller and computer to control actuator operation. Industrial camera mounted in the detection of car, Detection car driven by a stepper motor, Single-chip microcomputer control the stepper motor for the distance, direction and speed based on the receipt of control commands sent by the host computer. The whole system was controlled by SCM and PC machine via serial communication[2]. As shown in the figure 2.

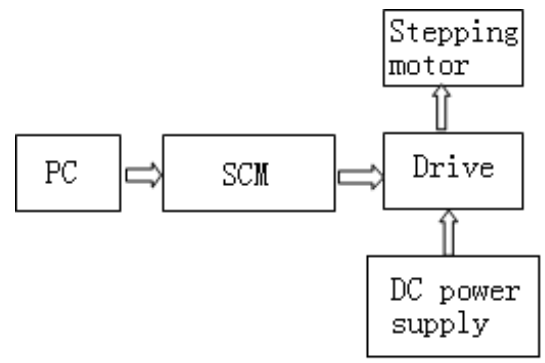

Fig 2 System components

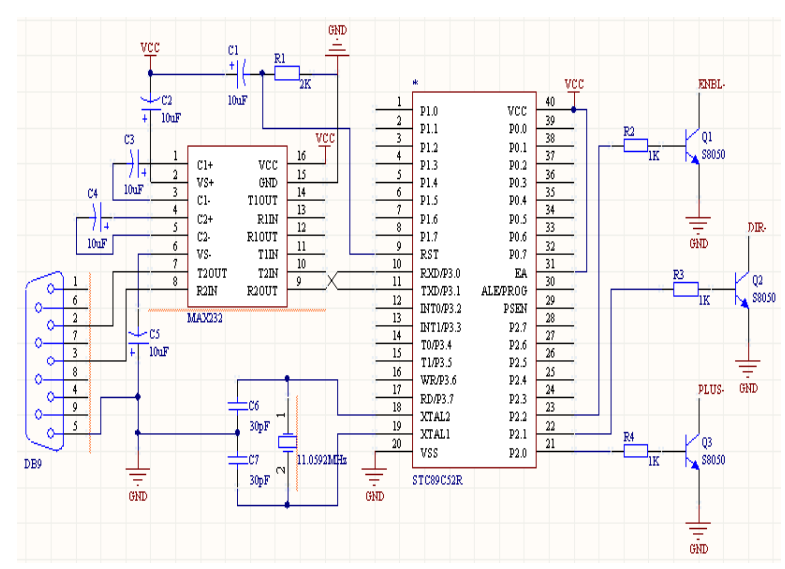

Fig 3 Control circuit
Figure 3 is the control circuit which is based on STC89C52 single-chip microcomputer between serial communication and stepper motor, which is applied for serial communication mode. Due to the PC serial logic is RS- 232C level, But STC89C52RC serial logic is TT L level, so they can not be directly connected, only need for a level conversion. In this system, the MA X232 as the level conversion device, STC89C52RC single-chip computer and PC serial communication interface circuit as shown in figure 4. Through MAX232 conversion from RS - 232C serial signal to connect to the DB9 socket with external PC connection, we can establish a RS- 232C communication interface.

The pulse, swerve and signal which come out from P2.0, P2.1, P2.2 of P2 will be transferred to step motor driver.

\section{IMAGE PROCESSING TECHNOLOGY AND SYSTEM}

The image of the steel tape will be collected and transmitted to the host computer by the image acquisition system, each picture will be processed by the host computer 's software, as the steel tape has much more types, different colors, different line structure, the device in the image processing aspects need to solve the problem : using the correct method of detection the standard and the tested steel tape meter the groove center position, so that the measuring accuracy of the system is guaranteed.

\section{A. Image processing}

The collection of images can not directly identify with the identification procedures, firstly, it need gray pretreatment[3], Gray scale image is R, G, B 3 components as a special color image, the 1 image points changing range has 255 kinds, so in the digital image processing, in order to make the following images' calculation less, we can change the first images in various formats into gray image. Gray images' description is the same as color image which reflects the whole image the whole and local chrominance and luminance levels of the distribution and characteristics. Gray scale image processing can be achieved by two methods .

The first method: calculate each pixel point R, G, B three component average value, then this average value is assigned to the pixel of the three component.

The second method: in the YUV color space, Y component represents the point of brightness, the value reflects the luminance level, according to the RGB and YUV color space of the relationship can be established with $R \mathrm{G}$, the brightness of $\mathrm{Y}$, B three color components corresponding relationship:

$$
Y=0.3 R+0.59 G+0.11 B
$$

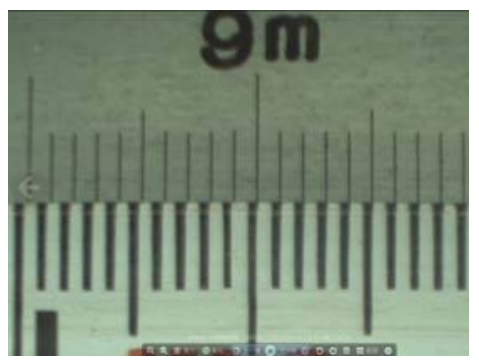

Fig 4 The original 
With the brightness value expression of image gray level, Figure 4 shows the original, figure 5 is the gray after processing.

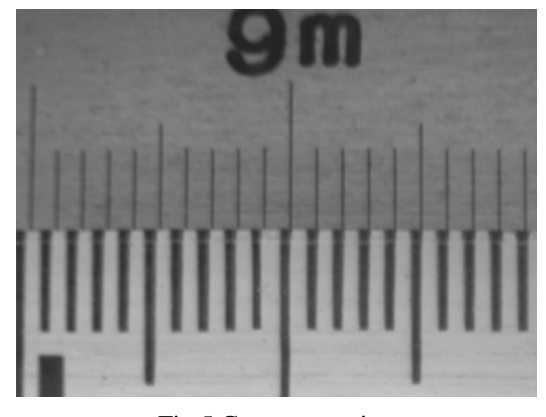

Fig 5 Gray processing

\section{B. Partition image of binarization processing}

Binary image processing is that the point of image gray level is set to 0 or 255 , that means the images clearly are shown the effect of black and white[4]. That means the 256 luminance level gray image can still reflect the global and local features of the binary image through appropriate threshold selection. In digital image processing, binary image plays a very important role, especially in the practical image processing. There are much more using binary image processing to achieve the structure system, if you have binary image processing and analysis, firstly, you have the gray value of the two images to get two binary images, when it is favor to make the image for further processing, image collection properties are only bond with the pixel values of 0 or 255 points, not involving the pixel value to multistage, thus the processing becomes simple, and the processing of data and compression become very small.

The tested steel ruler scale line and the background color is various, well, the standard steel tape color is fixed, so it is necessary seperate a standard ruler and tested ruler in different region to select a different threshold binarization processing, the binary image of partition processed as shown in figure 6:

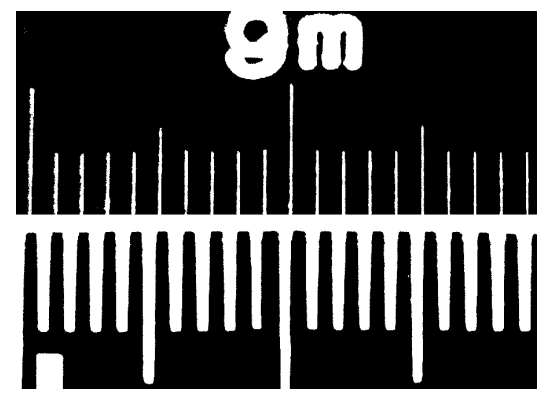

Fig 6 Binary image

\section{Detection of the groove center}

Considering the steel tape line is very exiguous, it is much easier to guarantee the precision compared the test groove center line to the edge detection, After the image was processing by binary and amplify it in $\mathrm{X}$ direction, then we can get data acquisition, when data is collecting, Click on the measured line, software will search the boundary line, automatically, and calculate the line center in $\mathrm{X}$ to pixel coordinates on the screen.

\section{Calculation of image pixel equivalent}

Pixel equivalent is that the corresponding conversion relationship between the unit pixel and the actual length[5]. That means to confirm each pixel representing actual length value in image measurement system, In the actual verification, due to the CCD field of view within the standard steel tape, so the calculation of standard steel tape length $\mathrm{L}$ corresponding to the pixel number $\mathrm{N}$, pixel equivalent for:

$$
\beta=L / N
$$

\section{E. Calculation error}

Using software to calculate difference between the standard reticle and measured line of pixel $\mathrm{M}$, the scribe of the error values:

$$
\delta=M \beta
$$

\section{CONCLUSION}

Using the industrial video camera in the measured line collecting images with the partition type of Binary image and the groove center detection image processing technology, combined with mechanical and electrical integration control technology, it realizes the automatic test of steel tape. Test results are automatically stored into the database or output statements, which improves the efficiency and precision of calibration.

\section{REFERENCE.}

[1] Xu Jun, Based on machine vision inspection technology of steel tape automatic verification device. Measurement technology, 2009 ( 11): 50-53.

[2] Xu Hongbin, Du Xiangbin. Design of stepping motor control system [J]. Journal of Chongqing Institute of Technology, 2008.22 ( 5): 32-34.

[3] Ruan Qiuqi. Digital image processing [M ] . Beijing: Publishing House of electronics industry, 2005

[4] Li Mingliang, Chen Bin, Fang Jie . Digital identification technology on steel tape self-verifying system [J]. Mechanical \& Electrical Engineering, 2008,25 (9): 62-64.

[5] Li Mingliang, Chen Bin, Fang Jie. The Application of screw size measurement by Image processing [J]. Fujian computer, 2010 ( 2): 15-16.

[6] Gao Weixin, Mu Xiangyang, Tang Nan. Based on image processing of weld porosity. Micro computer information to detect [J], 2010,26 ( 2-2 ): 33-35. 